\title{
Patient transfers during hospitalization: An examination of intra facility patient locations using network analysis
}

\author{
Joshua Sadowski*1, Mark Luetkemeyer ${ }^{1,2}$, Areeba Kara ${ }^{2}$ \\ ${ }^{1}$ Indiana University Health Academic Health Center, United States \\ ${ }^{2}$ Indiana University School of Medicine Department of Medicine, United States
}

Received: January 12, 2020

DOI: $10.5430 /$ jha.v9n1p43
Accepted: February 20, 2020

Online Published: February 27, 2020

\begin{abstract}
Patient movements following hospitalization are difficult to track. In a large Midwestern academic institution, we analyzed data using network statistics for patients discharged by the hospitalist service between June 2016-June 2018. We retrieved all major patient movements logged in the patient throughput management system following admission. The 4,869 patients discharged by the hospitalist service during the study period experienced 6,832 movements. The mean was 1.4 movements per patient while the maximum was 8 . Most patients $(72.3 \%$ ) moved once following hospitalization while $27.7 \%$ moved more than once. The predominant movement type was downgrades which comprised $51.8 \%(\mathrm{n}=3,543)$ of all movements. Lateral movements were the next most common $(25.9 \%, \mathrm{n}=1,771)$. Network statistics revealed progressive care units to be central to patient flow across the system. Transfers following hospitalization are common. Visualizing these transfers using network statistics may provide valuable insights to enhance patient safety.
\end{abstract}

Key Words: Patient safety, Hospitalists, Movement, Networks

\section{INTRODUCTION}

Hospitalization exposes patients to multiple transitions. Many of these transitions, including the handoff between the inpatient and outpatient setting, are known to represent a period of heightened risk. ${ }^{[1]}$ Similarly, end-of-shift handoffs between nurses and clinicians pose risks which may be attenuated by structured communication. ${ }^{[2]}$ Intra-hospital transitions following admission to the hospital (e.g. transfers between inpatient units) are also susceptible to breakdowns in communication, impact patient safety and pose additional distinct challenges. ${ }^{[3]}$ Transfers between inpatient units in a hospital are difficult to track. To quantify intra-hospital transfers, we undertook the novel approach of describing inpatient movements through exploratory network analysis.

\section{METHODS}

\subsection{Setting and population}

This work was conducted at a large, urban, tertiary care center in the Midwestern United States. The hospital has 500 beds, serves as a Trauma 1 center and discharges 22,000

*Correspondence: Joshua Sadowski; Email: jsadowski@iuhealth.org; Address: Indiana University Health Academic Health Center, United States. 
patients annually of which $40 \%$ are seen by the hospitalist service. Data for patients discharged by the hospitalist service over two years (June 2016 - June 2018) were analyzed.

\subsection{Data sources and analysis}

The institution utilizes a specialized Electronic Medical Record (EMR) system, to track patient movement. ${ }^{[4]}$ The system does not utilize any specific geo-locating devices and captures patients' location through the EMR. This ensures that only major location changes such as inter unit transfers are represented. We examined patient movements which followed the initial admission and excluded the emergency room, labor and delivery, diagnostic, procedural and "unknown" unit placements from the dataset.

Network analysis is used in a variety of disciplines to understand how individual entities interrelate within a system. Data were formatted for network analysis, creating an edge and node list. Each inpatient unit was designated as a "node" and each movement between nodes was counted as a transfer occurrence, creating an "edge" list. This revealed the relationships between units within the hospital and enabled us to construct network statistics to describe these relationships.

Basic network statistics were calculated for their descriptive nature and ease of interpretation. These metrics include a hub score which is a measure of how often the node receives traffic within a network. The hub score describes the influx of patients arriving to a particular unit and reflects how often other units utilize that unit for sending patients on to. An authority score was also calculated which examines the amount of outgoing traffic from an individual node within the network. In the context of our work, it reflects patients being transferred from one location to others. Eigenvector centrality was used to determine the interconnectedness of nodes which measures the importance of a single unit to the overall placement of patients throughout the hospital. ${ }^{[4]}$

An upgrade was defined as patient movement from a unit that provides a lower level of care to one that provides a higher level of care (medical surgical unit to progressive or intensive care). Downgrades reflected the reverse while lateral movements were movements within a unit or between units that provide similar levels of care.

\section{RESUlts}

The hospitalist service discharged 4,869 patients during the study period. These patients experienced 6,832 movements following admission for an average of 1.4 movements per patient. The maximum number of movements was 8 . Most patients $(72 \%)$ moved once following hospitalization while $27 \%$ moved more than once. Most movements $(86 \%, \mathrm{n}=$
5,885) occurred during the 7:00 a.m. to 7:00 p.m. shift.

There were 383 unique "paths" a patient could take from the time of admission to the inpatient unit to discharge. The predominant movement type was downgrades which comprised $51.8 \%(n=3,543)$ of all movements. Lateral movements were the next most common $(25.9 \%, \mathrm{n}=1,771)$ while the remaining movements were upgrades $(22.2 \% \mathrm{n}=1,518)$. Figure 1 represents these movements visually.

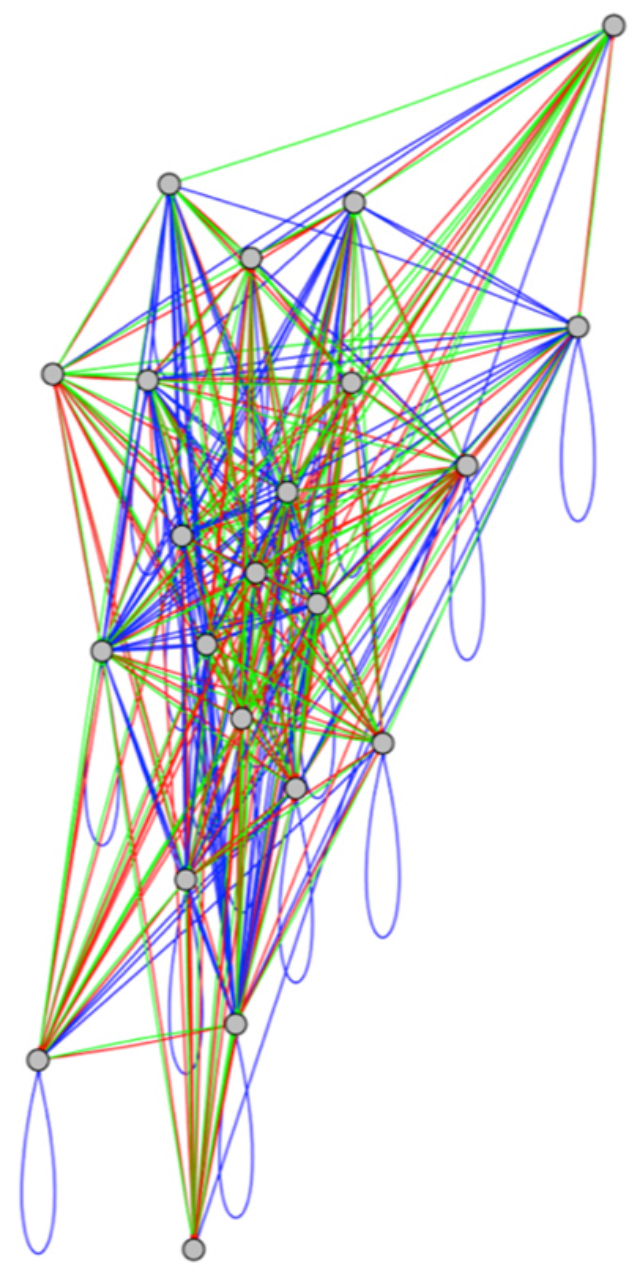

Figure 1. Network representation of patient movements between units

Red lines $=$ upgrade, blue lines $=$ lateral movements, green lines $=$ downgrades

Table 1 details the overall network statistics, illustrating the strength in the relationship between certain units that share patients throughout them within the facility. PCU3 was noted to have the highest hub and centrality scores indicating the high volume of patients this unit receives and its importance in influencing flow across the system. Conversely Med Surg unit 9 has the highest authority score reflecting the volume 
of patients that are transferred out from that location.

Table 1. Network statistics describing patient movements

\begin{tabular}{|c|c|c|c|}
\hline Node Name & Hub & Authority & Centrality \\
\hline Medical/Surgical 1 & 0.050 & 0.124 & 0.138 \\
\hline Medical/Surgical 2 & 0.035 & 0.220 & 0.185 \\
\hline Medical/Surgical 3 & 0.014 & 0.082 & 0.068 \\
\hline Medical/Surgical 4 & 0.021 & 0.060 & 0.066 \\
\hline Medical/Surgical 5 & 0.016 & 0.072 & 0.066 \\
\hline Medical/Surgical 6 & 0.257 & 0.520 & 0.499 \\
\hline Medical/Surgical 7 & 0.036 & 0.134 & 0.118 \\
\hline Medical/Surgical 8 & 0.050 & 0.140 & 0.147 \\
\hline Medical/Surgical 9 & 0.230 & 1.000 & 0.779 \\
\hline Medical/Surgical 10 & 0.106 & 0.742 & 0.549 \\
\hline Medical/Surgical 11 & 0.085 & 0.529 & 0.422 \\
\hline PCU 1 & 0.220 & 0.096 & 0.321 \\
\hline PCU 2 & 0.113 & 0.034 & 0.130 \\
\hline PCU 3 & 1.000 & 0.197 & 1.000 \\
\hline ICU 1 & 0.130 & 0.278 & 0.345 \\
\hline ICU 2 & 0.091 & 0.163 & 0.202 \\
\hline ICU 3 & 0.062 & 0.085 & 0.124 \\
\hline ICU 4 & 0.024 & 0.017 & 0.040 \\
\hline ICU 5 & 0.021 & 0.008 & 0.029 \\
\hline ICU 6 & 0.046 & 0.052 & 0.091 \\
\hline ICU 7 & 0.043 & 0.078 & 0.093 \\
\hline ICU 8 & 0.013 & 0.017 & 0.024 \\
\hline
\end{tabular}

Note. PCU = Progressive Care Unit; ICU = Intensive Care Unit

\section{Discussion}

Inter-unit transfers comprise up to a quarter of the critical safety incidents related to handovers. ${ }^{[5]}$ Our work demonstrates that most patients admitted to a hospital medicine service are exposed to these risks with the majority experiencing at least one movement. While these figures and the large number of unique paths that these movements represent may seem overwhelming, visualizing them in the network format may help us to ask questions, generate hypotheses and inform our interventions. For example, visualizing the data for our hospitalist service offered us several new insights: we noted the central importance of the medical progressive unit (PCU 3) to the overall flow of patients and noted an unexpected number of lateral and intra-unit transfers. These realizations have prompted us to further study transfers to and from the medical PCU and the phenomenon of intra-unit transfers.
By understanding the interrelationships between units, hospitals may be able to better plan for the utilizations of their teams, enhance bed management, and understand patient flow. Handoff processes are more likely to be successful if they can be customized to the relationships between units. Adverse events during downgrades are often related to poor communication and nursing discomfort with caring for specific populations. ${ }^{[3]}$ Using this data to create targeted education and interventions for the two connected units may decrease patient harm related to transfers between these units.

Networks can guide teams to examine harm events, create better care paths, and reallocate team members to maximize patient safety and experience by examining how patients move through the facility. Patients experiencing unplanned transfers to the intensive care unit have high mortality rates. ${ }^{[6]}$ Rapid response teams improve mortality by recognizing and interrupting the trajectory of a deteriorating patient but may be underutilized afterhours. ${ }^{[7,8]}$ Evaluating patterns of upgrades and their diurnal variation may help focus the attention of rapid response teams and develop monitoring systems to anticipate the needs of these patients.

Many large hospitals have disease or pathology specific inpatient units however patients with specific conditions best served on a particular unit may be dispersed over the entire hospital. While the clinical microsystem and unit-based interventions are critically important to improving outcomes, ${ }^{[9]}$ our work suggests that patients' care pathways are rarely straightforward. Analyzing the network in relationship to specific disease states may help us both assess any differences in outcomes related to mismatches between patient need and patient placement, but also to create a map of our patient needs. Similarly, while there may be benefits of geographically localizing or cohorting hospitalists to a single unit, our findings highlight the complex challenges of balancing cohorting with continuity of care. ${ }^{[10]}$

While upgrades and downgrades are clinically intuitive, we were surprised by the large number of lateral movements, many of which were within the same unit. Each time a patient is transferred, valuable time is lost while the room is prepared for the next occupant. Our work does not enable us to explain the underlying reasons for lateral transfers, however given the large volume of such movements, further research exploring the role of staffing and unit design may be warranted.

This work has limitations. It is a single center's experience and our findings may not be reproducible. We did not examine movements between procedural and diagnostic areas and therefore these numbers underestimate the number of handoffs during a patient's hospital stay. We cannot comment 
on the pathways for patients admitted to specialties other than hospital medicine. We were unable to extrapolate paths specific to patient diagnoses or risk stratify the population.

A patient's path through a hospitalization is complex and susceptible to communication breakdowns. Intra-hospital transfers are common and may be underestimated in their contribution to harm. Visualizing patient movements using network analysis may enable institutions to identify opportunities for improvement and help patients journey safely.

\section{Conflicts of InTEREST Disclosure}

The authors declare they have no conflicts of interest.

\section{REFERENCES}

[1] Krumholz HM. Post-hospital syndrome-an acquired, transient condition of generalized risk. New Engl J Medicine. 2013; 368: 100-102. PMid: 23301730. https://doi.org/10.1056/NEJMp1212324

[2] Halm MA. Nursing Handoffs: Ensuring Safe Passage for Patients. Am J Crit Care. 2013; 22: 158-162. PMid: 23455866. https: //doi.org/10.4037/ajcc2013454

[3] Ong MS, Biomed EM, Coiera E. A Systematic Review of Failures in Handoff Communication During Intrahospital Transfers. Jt Comm J Qual Patient Saf. 2011; 37: 274-AP8. https://doi.org/10.101 6/S1553-7250(11) 37035-3

[4] Ognyanova K. Network analysis with R and igraph: NetSci X Tutorial. 2016. Available from: www.kateto.net/networks-r-igr aph

[5] Pezzolesi C, Schifano F, Pickles J, et al. Clinical handover incident reporting in one UK general hospital. Int J Qual Health C. 2010; 22: 396-401. PMid: 20709704. https://doi.org/10.1093/intqhc /mzq048
[6] Mochizuki T. Mortality among unplanned intensive care unit transfer cases. Critical Care Medicine. 2012; 40: 193. https ://doi.org/ 10.1097/01.ccm.0000424987.77547.fd

[7] Maharaj R, Raffaele I, Wendon J. Rapid response systems: a systematic review and meta-analysis. Crit Care. 2015; 19: 1 15. PMid: 26070457. https://doi.org/10.1186/s13054-015-0973-y

[8] Churpek MM, Edelson DP. In search of the optimal rapid response system bundle. J Hosp Med. 2015; 10: 411. PMid: 25772513. https://doi.org/10.1002/jhm. 2346

[9] O'Leary KJ, Johnson JK, Manojlovich M, et al. Use of Unit-Based Interventions to Improve the Quality of Care for Hospitalized Medical Patients: A National Survey. Jt Comm J Qual Patient Saf. Epub ahead of print 2017. PMid: 29056177. https://doi.org/10.101 $6 / j \cdot j c j q .2017 .05 .008$

[10] Kara A, Johnson CS, Hui SL, et al. Hospital-Based Clinicians' Perceptions of Geographic Cohorting: Identifying Opportunities for Improvement. Am J Med Qual. 2018 May/Jun; 33(3): 303-312. PMid: 29241347. https : //doi.org/10.1177/1062860617745123 